\title{
Characteristics of Patients Seeking and Proceeding with Non-Surgical Facial Aesthetic Procedures
}

This article was published in the following Dove Press journal:

Clinical, Cosmetic and Investigational Dermatology

\author{
Sylvia PB Ramirez (D) \\ Gunther Scherz' \\ Helen Smith ${ }^{2}$ \\ 'Cutis Medical Laser Clinics Pte Ltd, \\ Singapore; ${ }^{2}$ Family Medicine and Primary \\ Care, Lee Kong Chian School of \\ Medicine, Nanyang Technological \\ University Singapore, Singapore
}

\begin{abstract}
Purpose: Identifying predictors for patients' readiness to receive non-surgical facial aesthetic treatments facilitates the physician's understanding of the patient's goals and expectations. This paper aims to identify clinical and demographic characteristics of patients who proceed with non-surgical facial aesthetic procedures in Singapore.

Patients and Methods: Using data from electronic patient health records, authors examined clinical and demographic characteristics of 624 Asian and Caucasian patients who sought treatment in a 12-month period and who had minimum follow-up of 1 year. Variables examined included age, race/ethnicity, gender, prior treatment, and attitudes and motivation for seeking treatment. Univariate and multivariate analyses of factors associated with proceeding with the treatment plan were evaluated using chi-square and logistic regression analyses.
\end{abstract}

Results: Approximately $88 \%$ of patients who sought consultation proceeded with treatment. The majority were older than 40 years of age, were female and received prior treatments. Notable is the high frequency of rejuvenation rather than correction as the clinical outcome goal. There were slightly more Caucasians than Chinese patients but the racial distribution allowed the identification of differences between the two groups. Chinese patients were younger and more likely to seek correction or more obvious changes as compared to Caucasian patients. On multivariate analyses, powerful predictors for proceeding with noninvasive facial treatments included a treatment goal of rejuvenation rather than correction, an expectation of an immediate result, and prior aesthetic treatments.

Conclusion: This study is the first to compare race/ethnic differences in factors that influence treatment with non-invasive aesthetic procedures. This is also the first to identify demographic, clinical and motivational characteristics associated with actual treatment with non-invasive facial procedures. Further research on a systematic approach to defining a patient's motivation for receiving treatment, as well as research identifying which patients are more likely to have positive clinical outcomes are warranted.

Keywords: predictors, motivations, non-invasive, rejuvenation, race, ethnicity

\section{Introduction}

There has been an exponential increase in global demand for cosmetic procedures. ${ }^{1}$ In its annual report, the American Society for Aesthetic Plastic Surgery reported a 16.5-billion-dollar expenditure in 2018 alone, and a $228 \%$ increase in nonsurgical facial aesthetic (NSFA) procedures from 2000 to 2018. Furthermore, in the corresponding period, $95 \%$ of all cosmetic treatments undertaken were for NSFA treatments with a $5 \%$ decrease in surgical procedures. ${ }^{1}$

Cosmetic procedures are "life-enhancing" rather than "life-saving" and therefore the characteristics, motivation and goals of patients seeking cosmetic
Correspondence: Sylvia PB Ramirez Cutis Medical Laser Clinics Pte Ltd, 9 Scotts Road, Scotts Medical Center \#0807, 228210, Singapore

Tel +6590400113

Email ramirezs224@gmail.com 
procedures likely differ from patients in other fields of medicine. ${ }^{2}$ To be able to conduct an effective and focused consultation process, doctors offering aesthetic procedures need a greater understanding of characteristics, sociodemographic factors, motivations, expectations and treatment readiness of their patients, as these may inform treatments to be proposed in the short- and long-term treatment plans.

To date, most published studies focus on predictors of, and openness to traditional plastic surgery rather than NSFA procedures. Some evaluate the clinical, demographic and psychological issues associated with attitudes towards and acceptance of cosmetic procedures. ${ }^{3-6}$ They do not address characteristics of patients who progress to actual treatment with cosmetic procedures.

Published literature on traditional plastic surgery has also addressed the psychosocial factors among those seeking surgery. High prevalence of psychopathology among those seeking plastic surgery have been reported. ${ }^{7,8}$ In one study, $47.7 \%$ of patients seeking plastic surgery had "mental" disorders as defined by ICD-10 medical codes" compared with a prevalence of $18.5 \%$ within the general US population. ${ }^{10}$ Another study described a 6 -fold higher prevalence of body dysmorphic disorder among those seeking cosmetic surgery as compared to the general population. ${ }^{11}$ In addition, one prospective study of adolescent females identified mental health psychological conditions, including depression, anxiety, history of self-harm, and suicide attempts as strongly predictive of plastic surgery. ${ }^{12}$ In another study, a significant association between high-risk behaviors (including alcohol and drug abuse intake and partner abuse) and cosmetic surgery was observed. ${ }^{13}$ It is not known whether similar psychologic and psychosocial factors are associated with NSFA procedures.

Despite the high demand for non-surgical facial aesthetic procedures, only two studies have examined patients' motivations for seeking NSFA treatments. ${ }^{14,15}$ One study identified demographic (female gender, middleaged, higher income levels) and personal characteristics (increased attention to skin health) as predictors for consultation for non-surgical treatments. Procedure cost was reported as a barrier to treatment by $40 \%$ of patients. ${ }^{15}$ In the second study, a questionnaire was used to identify patient motivations for treatments. ${ }^{14}$ The study identified improvements in confidence and well-being as primary motivators for seeking cosmetic procedures. Unfortunately, these studies from the United States failed to address differences between racial or ethnic groups.
Given the rapid expansion of non-surgical facial aesthetics globally, a greater understanding of how patient characteristics and motivational factors differ among ethnic groups is becoming increasingly important, and factors that influence actual conduct of treatment need to be examined.

Recognising these gaps in knowledge and research about NSFA procedures, this study describes the clinical and demographic characteristics of patients seeking nonsurgical facial aesthetic procedures in a private clinic in Singapore, and their readiness to proceed. Understanding clinical and motivational characteristics of patients may allow physicians to be more effective when addressing patient concerns during the consultation process.

\section{Patients and Methods}

\section{Study Population}

Patients included in the study were new patients (incident) to a Singapore-based clinic between January 2014 to December 2015 with a minimum of 1-year follow-up. Included patients were adults ( $\geq 21$ years) and those of Caucasian and Chinese ethnicity. Other ethnic groups were excluded because these were very heterogeneous and constituted a small proportion $(3.7 \%)$ of the clinic population.

\section{Data Collection}

The data used in these analyses came from the form completed by each patient prior to their first consultation, which is saved in an electronic data system and utilized for clinical care.

Specific data items analyzed included age; gender; race (Chinese versus Caucasians); number of facial aesthetic concerns ( 1 versus 2 or more); prior treatment with botulinum toxin, dermal filler, laser or plastic surgery (yes versus no for each); self-reported anxiousness for nonsurgical facial aesthetic treatments (none versus anxiety from side-effects, pain, dissatisfaction with results and others); self-reported openness to receive botulinum toxin, fillers, laser procedures (yes versus no for each); having favorable skin habits (regular sunscreen and nonsmoker versus all others); reason for non-surgical facial aesthetic treatments (rejuvenation- self-reported goal of 1) feeling slightly younger and refreshed, 2) feeling less selfconscious and/or 3) feeling more confident versus correction- self-reported goal of 1) looking significantly younger, 2) correcting a physical feature, 3) wanting to look completely different), 4) appearance is top priority, 
and "People comment on my appearance"; vanity score (frequency of self-checking in a mirror of $\leq 10$ times versus $>10$ times per day); self-reported motivation (intrinsicresponding as "I want to do it myself" and/or "Look the way I feel" versus extrinsic-responding as "Preparing for a milestone", "Dealing with a life-changing event", "To please others"); expectations of outcomes (immediatevisible improvement $\leq 30$ days versus progressive-visible outcomes over time); patient-reported self-esteem (positive-responding "very happy" or "happy" about self versus negative- "neutral", "unhappy" and "very unhappy" about self).

The accuracy of data extraction was confirmed by the repeated extraction of a $10 \%$ random sample of data. Agreement exceeded 99\% and was considered acceptable.

\section{IRB Approval}

The study was conducted in accordance with the Declaration of Helsinki. The Nanyang Technological University Lee Kong Chian School of Medicine served as the Institutional Review Board for this study (IRB2019-09-009). Given that data for analyses were collected as part of patient care responsibilities, formal IRB exemption was given by the review board. It should be noted that each patient's written consent is routinely sought in clinic for the conduct of record-based research. Only deidentified data were utilized in the analyses, and only the investigator and members of the clinical team had direct access to patient-level data.

\section{Data Analyses}

Frequency distributions were calculated for the variables as described above. Continuous variables were categorized and described as frequencies and percentages. To further understand associations between population characteristics, Chi-square analyses were undertaken, only those associations that were statistically significant are presented.

Predictors of proceeding with treatment, which was defined by spending above the consultation fee in the first year ( $\mathrm{Y}$ vs $\mathrm{N}$ ) were evaluated. The univariate or unadjusted associations between clinical or demographic factors (transformed into categorical variables) and proceeding with treatment plan ( $\mathrm{Y}$ vs $\mathrm{N}$ as reference) were examined using univariate logistic regression models. ${ }^{16}$ Multivariate logistic regression models were used to estimate the adjusted odds ratios for proceeding with treatment within the 1-year follow-up period after adjusting for other independent variables. The base model included variables with a $\mathrm{p}<0.05$ on univariate analyses. ${ }^{16}$ Stepwise logistic regression was performed as a sensitivity analyses, which added other potential explanatory variables while noting the change in statistical significance of the estimated odds ratios. ${ }^{16}$

SPSS Version 25 was used for all statistical analyses. Statistical significance was defined as $\mathrm{p}<0.05$.

\section{Results}

Clinical and demographic features of the study population are presented in Table 1 . Of the 624 patients, $53.1 \%$ were older than 40 years, $86.9 \%$ were female and there were slightly more Caucasians than Chinese (51.6\% vs $48.4 \%$, respectively). The majority of patients noted 2 or more complaints in their consultation form (70.7\%), and over $70 \%$ had prior treatment with botulinum toxin, dermal fillers, lasers and/or plastic surgery. Favorable lifestyle (regular use of sunscreen and no smoking) was observed in $45 \%$ of patients seeking treatment. Over $88 \%$ proceeded with one or more components of the treatment plan recommended during consultation.

With regards to attitudes to treatments, $99.8 \%$ of patients expressed openness for various procedures, with $89.7 \%$ willing to consider botulinum toxin, and $89 \%$ treatment with dermal fillers. Even more patients were willing to consider laser procedures $(93.8 \%)$, as well as other treatments including ultrasound, thread lifts and microneedling (96.6\%). The main goal for treatment was reported as rejuvenation by $87.6 \%$ of patients, whereas $26.2 \%$ reported correction as their goal (13.8\% reported a goal of both rejuvenation and correction). Self-checking in a mirror more than 10 times a day was reported by $3.9 \%$ of patients. A majority of patients $(88.2 \%)$ reported an "organic" reason for seeking treatment ("wanting to look the way they feel", see Materials and Methods). Patients more frequently sought immediate $(68.2 \%)$ rather than progressive or gradual results of aesthetic treatments (31.8\%). Positive self-esteem rather than neutral or negative self-esteem was reported by $74.8 \%$ of patients.

Patient demographic and clinical characteristics were cross-tabulated to further understand patients who sought consultation for non-invasive facial aesthetic procedures. There was a significant association between race/ethnicity and age of presentation to the clinic (Table 2, $\mathrm{p}<0.0001$ ). Caucasians were overrepresented in the age groups between 30-39 (54\% Caucasian vs 46\% Chinese) and 40-49 years $(56.7 \%$ Caucasian vs $43.3 \%$ Chinese), 
Table I Characteristics of Patients Presenting for the First Time to Clinic $(N=624)$

\begin{tabular}{|c|c|}
\hline Characteristics & Number (\%) \\
\hline Proceeded with treatment & $552(88.5)$ \\
\hline \multicolumn{2}{|l|}{ Age Distribution $(N=624)$} \\
\hline$<30$ & $78(12.5)$ \\
\hline $30-<40$ & $213(34.1)$ \\
\hline $40-<50$ & $233(37.3)$ \\
\hline 50 and above & $100(16.02)$ \\
\hline \multicolumn{2}{|l|}{ Gender $(N=624)$} \\
\hline Male & $82(13.1)$ \\
\hline Female & $542(86.9)$ \\
\hline \multicolumn{2}{|l|}{ Race $(N=624)$} \\
\hline Chinese & $308(49.4)$ \\
\hline Caucasian & $316(51.6)$ \\
\hline \multicolumn{2}{|l|}{ Number of complaints $(\mathrm{N}=624)$ : } \\
\hline 1 & $183(29.3)$ \\
\hline 2 or more & $44 \mid(70.7)$ \\
\hline Prior Botox $(\mathrm{N}=618)$ & $261(42.2)$ \\
\hline Prior Fillers $(\mathrm{N}=618)$ & $144(23.3)$ \\
\hline Prior Laser/cosmetic procedure $(\mathrm{N}=618)$ & $308(49.8)$ \\
\hline Prior Plastic surgery $(N=613)$ & $61(10.0)$ \\
\hline Prior Botox or Fillers or Laser $(N=619)$ & $429(69.3)$ \\
\hline Prior Botox or Fillers or Laser or Plastic Surgery $(N=619)$ & $435(70.3)$ \\
\hline No prior treatment $(\mathrm{N}=619)$ & $184(29.7)$ \\
\hline Favourable Lifestyle (no smoking, regular sunscreen) $(\mathrm{N}=616)$ & $277(45.0)$ \\
\hline Openness to treatment: Botox $(\mathrm{N}=624)$ & $560(89.7)$ \\
\hline \multicolumn{2}{|l|}{ Openness to treatment: Filler $(\mathrm{N}=624)$} \\
\hline Yes & $556(89.1)$ \\
\hline No & $68(10.9)$ \\
\hline Openness to treatment: Laser $(\mathrm{N}=624)$ & $585(93.8)$ \\
\hline Openness to other treatments $(\mathrm{N}=624)$ & $603(96.6)$ \\
\hline Openness to Botox, fillers, lasers, or other treatments $(N=624)$ : Yes & $723(99.8)$ \\
\hline Level of Anxiousness ( $N=604)$ : None & $420(69.5)$ \\
\hline \multicolumn{2}{|l|}{ *Reason for doing Procedure (588): Rejuvenation (I-3) } \\
\hline Yes & $515(87.6)$ \\
\hline No & $73(12.4)$ \\
\hline \multicolumn{2}{|l|}{ Correction (4-9) } \\
\hline Yes & $154(26.2)$ \\
\hline No & $434(73.8)$ \\
\hline \multicolumn{2}{|l|}{ **Vanity Score (frequency of self-check) $(N=57 \mathrm{I})$ : } \\
\hline Low $(\leq 10$ times per day) & $549(96.1)$ \\
\hline High (>10 times per day) & $22(3.9)$ \\
\hline
\end{tabular}


Table I (Continued).

\begin{tabular}{|l|c|}
\hline Characteristics & Number (\%) \\
\hline$* * *$ Motivations $(\mathrm{N}=586):$ & $517(88.2)$ \\
I Intrinsic & $69(11.8)$ \\
2 Extrinsic & \\
\hline$* * * *$ Expectations $(\mathrm{N}=575):$ & $392(68.2)$ \\
I Immediate (results $\leq 30$ days) & $183(31.8)$ \\
2 Progressive (results $>30$ days) & \\
\hline$* * * *$ Self-Esteem $(\mathrm{N}=563):$ & $421(74.8)$ \\
I Positive & $142(25.2)$ \\
2 Neutral or Negative & \\
\hline
\end{tabular}

Notes: *Reason for doing Procedures: a) Rejuvenation defined as any one of the following responses - I: I want to appear younger and refreshed, 2: I want to feel less selfconscious about my appearance, 3: I want to feel more confident in my personal and professional relationships. b) Correction defined as one of the following responses, 4 : I want to look significantly younger, 5: I want to correct a physical feature, 6: I want to look completely different, 7: People comment on my appearance, 8: My appearance is one of my top priorities, 9: Others. Variable classified as either rejuvenation or not seeking rejuvenation, and correction vs not seeking correction. **Vanity score defined as Low-frequency of self-check of 10 times or less in a day and high-frequency of self-check more frequent than 10 times in a day. ***Motivations defined as Intrinsic - I: I want to do it myself or I want to look the way I feel, Extrinsic 2: I am preparing for a milestone event and 3: I am dealing with a life-changing event, 4: I want to please my partner I friends/family. *****Expectations defined as Immediate-visible results seen in 30 days or less, Progressive-visible results seen in 6 months or progressively. *****Self-esteem based on response to "How have you felt over the past few weeks?" Positive - very happy or happy, Negative - neutral, unhappy and very unhappy.

Table 2 Age vs Ethnicity

\begin{tabular}{|l|c|c|c|c|c|c|}
\hline \multirow{2}{*}{ Race } & \multicolumn{5}{|c|}{ Age Group } & \multirow{2}{*}{ Chi-Square (p-value) } \\
\cline { 2 - 7 } & Less Than 30 Years & $\mathbf{3 0 - 3 9}$ Years & 40-49 Years & 50 Years and Above & Total & \\
\hline Chinese & $58(74.4)$ & $98(46.0)$ & $101(43.3)$ & $51(51.0)$ & $308(49.4)$ & $23.94(<0.000 I)$ \\
Caucasian & $20(25.6)$ & $115(54.0)$ & $132(56.7)$ & $49(49.0)$ & $316(50.6)$ & \\
Total & 78 & 220 & 247 & 102 & 624 & \\
\hline
\end{tabular}

whereas Chinese patients dominated the younger age group $(25.6 \%$ Caucasian vs $74.4 \%$ Chinese). At older ages, there were almost the same distribution of Caucasian and Chinese patients (49\% Caucasian vs 51\% Chinese in the age group 50 years and older).

With regards to motivation for treatment, across all age groups, a goal of rejuvenation was significantly more common than a goal of correction (between $80.8 \%$ and $92.2 \%$ of patients in all age categories), although a slightly more prevalent goal of "correcting a physical feature" was reported between the ages of 40-49 years (Table 3, 18.3\% vs $10.7-15.1 \%$ for other age groups, $\mathrm{p}=0.005$ ). Rejuvenation as a treatment goal was markedly more common regardless of race/ethnicity (Table 4), but the desire for correction was significantly higher among Chinese as compared to Caucasian patients $(19.3 \%$ of Chinese vs $10.1 \%$ of Caucasian patients, $\mathrm{p}=0.003$ ).

Prior treatment with one or more forms of non-invasive facial aesthetic procedures was common in all patients (Table 5), however, prior treatment was significantly more prevalent in Caucasian patients $(76.8 \%$ of Caucasians vs $63.5 \%$ of Chinese, $\mathrm{p}<0.0001$ ). As expected, female patients were significantly more likely to have had prior treatment as compared to male patients (data not shown, $73.6 \%$ of female vs $48.8 \%$ of male patients, $\mathrm{p}<0.0001)$.

\section{Predictors of Proceeding with Treatment Plan}

Over $88 \%$ of patients proceeded to treatment and univariate analyses were performed to explore associations between demographic and clinical characteristic and likelihood of proceeding with treatments (Table 6). There was no association with age or gender, but Caucasian race was significantly associated with proceeding with treatment with a $70 \%$ greater likelihood compared to a Chinese patient (OR: 1.7, 95\% CI: 1.04-2.8, $\mathrm{p}=0.0335)$. The odds of proceeding with treatment was increased by prior treatment (OR: 1.9, 95\% CI: $1.2-3.2, p=0.009)$ and an expectation of immediate improvement after treatment (OR: 1.7, 95\% CI: 1.0-2.9, $\mathrm{p}=0.043$ ). Patients reporting a degree of anxiety towards 
Table 3 Age vs Reason for Treatment

\begin{tabular}{|c|c|c|c|c|c|c|c|}
\hline & & \multicolumn{5}{|c|}{ Age Group } & \multirow{2}{*}{$\begin{array}{c}\text { Chi-Square } \\
\text { (p-value) }\end{array}$} \\
\hline & & $\begin{array}{c}\text { Less Than } 30 \\
\text { Years }\end{array}$ & $\begin{array}{l}30-39 \\
\text { Years }\end{array}$ & $\begin{array}{l}40-49 \\
\text { Years }\end{array}$ & $\begin{array}{c}50 \text { Years and } \\
\text { Above }\end{array}$ & Total & \\
\hline Reason for & Rejuvenation* & $59(80.8)$ & $189(92.2)$ & $188(86.2)$ & 79 (85.9) & $515(87.6)$ & $23.55(0.005)$ \\
\hline \multirow[t]{3}{*}{ Treatment } & Correction $* *$ & II (15.I) & $22(10.7)$ & $40(18.3)$ & $12(13.0)$ & $85(14.5)$ & \\
\hline & Other self-concerns & $20(27.4)$ & $31(15.1)$ & 25 (II.5) & $14(15.2)$ & $90(15.3)$ & \\
\hline & Total & 73 & 205 & 218 & 92 & 588 & \\
\hline
\end{tabular}

Notes: *Rejuvenation defined as any one of the following responses - I: I want to appear younger and refreshed, 2: I want to feel less self-conscious about my appearance, 3: I want to feel more confident in my personal and professional relationships. **Correction defined as one of the following responses - 4: I want to look significantly younger, 5: I want to correct a physical feature, 6: I want to look completely different, 7: People comment on my appearance 8: My appearance is one of my top priorities, 9: Others. Variable classified as either rejuvenation or not seeking rejuvenation, and correction vs not seeking correction.

Table 4 Ethnicity vs Reason for Treatment

\begin{tabular}{|l|c|c|c|c|}
\hline \multirow{2}{*}{ Reason for Treatment } & \multicolumn{3}{|c|}{ Ethnicity } & \multirow{2}{*}{ Chi-Square (p-value) } \\
\cline { 2 - 5 } & Chinese & Caucasian & Total & $19.34(0.003)$ \\
\hline Rejuvenation* & $235(83.9)$ & $280(90.9)$ & $85(14.5)$ & \\
Correction** & $54(19.3)$ & $31(10.1)$ & $90(15.3)$ & 588 \\
Other self-concerns & $50(17.9)$ & $40(13.0 \%$ & 308 & 58 \\
Total & 280 & & \\
\hline
\end{tabular}

Notes: *Rejuvenation defined as any one of the following responses - I: I want to appear younger and refreshed, 2: I want to feel less self-conscious about my appearance, 3: I want to feel more confident in my personal and professional relationships. **Correction defined as one of the following responses - 4: I want to look significantly younger, 5: I want to correct a physical feature, 6: I want to look completely different, 7: People comment on my appearance 8: My appearance is one of my top priorities, 9: Others. Variable classified as either rejuvenation or not seeking rejuvenation, and correction vs not seeking correction.

Table 5 Ethnicity vs Prior Treatment

\begin{tabular}{|c|c|c|c|c|c|}
\hline \multicolumn{2}{|c|}{} & \multicolumn{3}{|c|}{ Ethnicity } & $\begin{array}{c}\text { Chi-Square } \\
\text { (p-value) }\end{array}$ \\
\cline { 3 - 7 } & & Chinese & Caucasian & Total & \\
\hline Previously had Botox or Filler or Laser or Plastic surgery & Yes & $193(63.5)$ & $242(76.8)$ & $435(70.3)$ & $13.18(<0.0001)$ \\
& No & $111(36.5)$ & $73(23.2)$ & $184(29.7)$ & 619 \\
& Total & 304 & 315 & \\
\hline
\end{tabular}

the procedure were significantly less likely to proceed with treatment (OR: 0.6, 95\% CI: 0.4-1.0, p=0.045) as were patients with a high vanity score (OR: 0.3, 95\% CI: $0.1-0.8, \mathrm{p}=0.02$ ).

To examine the simultaneous effect of multiple variables on proceeding to treatment we progressed to multivariate analyses, which included variables of statistical significance in univariate analyses and those that we considered clinically relevant (number of complaints, rejuvenation as a treatment goal, and self-esteem) (Table 7).

Age, gender, race/ethnicity, presence of anxiety towards the procedures, vanity score, and reported self-esteem were no longer associated with differences in the likelihood of proceeding with the treatment plan after adjusting for the simultaneous effects of other potential confounding factors. The three strongest predictors for proceeding with treatment were patient's prior treatment history, having a goal of rejuvenation for their procedures, and having an expectation for instant improvement after treatment. Prior treatment increased the likelihood of proceeding with treatment by almost three times (OR: 2.9, 95\% CI: $1.6-5.3, \mathrm{p}=0.001)$. In addition, a patient seeking facial rejuvenation rather than correction was 2.7 more likely to proceed with treatments (OR: 2.7, 1.2-5.8, $\mathrm{p}=0.014$ ). Finally, patients who expected immediate improvements were close to twice as likely to proceed with the treatment plan (OR: 1.9, 95\% CI: 1.1-3.5, $\mathrm{p}=0.033$ ). Interestingly, patients reporting two or more physical concerns on consultation were significantly less likely to proceed with the treatment plan (OR: 0.4, 95\% CI: $0.2-1.0, \mathrm{p}=0.04$ ).

Sensitivity analyses that added other available clinical and demographic factors did not change the findings of the multivariate model. 
Table 6 Univariate Association of Clinical and Demographic Factors and "Proceeded with Treatment"

\begin{tabular}{|c|c|c|c|c|}
\hline \multirow[t]{2}{*}{ Clinical and Demographic Characteristics } & \multirow[t]{2}{*}{ Odds Ratio } & \multicolumn{2}{|c|}{ 95\% Confidence Interval } & \multirow[t]{2}{*}{ p-value } \\
\hline & & Lower & Upper & \\
\hline Age ( $\geq 40$ vs ref: $<40 y r s)$ & 1.407 & 0.859 & 2.303 & 0.175 \\
\hline Female (vs ref: Male) & 1.542 & 0.804 & 2.958 & 0.192 \\
\hline Caucasian (vs ref: Chinese) & 1.714 & 1.037 & 2.833 & 0.035 \\
\hline Complaints (>2 vs ref: 1 ) & 0.659 & 0.367 & 1.182 & 0.162 \\
\hline Prior Treatment ( $\mathrm{Y}$ vs ref: $\mathrm{N})$ & 1.947 & 1.178 & 3.218 & 0.009 \\
\hline Anxious ( $\mathrm{Y}$ vs ref: $\mathrm{N}$ ) & 0.594 & 0.357 & 0.988 & 0.045 \\
\hline Favorable Lifestyle (Y vs ref: N) & 0.774 & 0.467 & 1.283 & 0.320 \\
\hline Rejuvenation ( $\mathrm{Y}$ vs ref: $\mathrm{N}$ ) & 1.850 & 0.954 & 3.588 & 0.069 \\
\hline Correction ( $\mathrm{Y}$ vs ref: $\mathrm{N}$ ) & 0.597 & 0.315 & 1.133 & 0.114 \\
\hline Vanity Score (High $\geq 10$ times vs ref: Low $<10$ times/day) & 0.315 & 0.119 & 0.837 & 0.020 \\
\hline Motivation (Internal vs ref: External) & 0.712 & 0.296 & 1.715 & 0.449 \\
\hline Expectation (Instant vs ref: Progressive) & 1.704 & 1.017 & 2.857 & 0.043 \\
\hline Self Esteem (Positive vs ref: Neutral or Negative) & 0.897 & 0.486 & 1.654 & 0.727 \\
\hline
\end{tabular}

Table 7 Multivariate Associations of Clinical and Demographic Characteristics and "Proceeded with Treatment"

\begin{tabular}{|c|c|c|c|c|}
\hline \multirow[t]{2}{*}{ Clinical and Demographic Characteristics } & \multirow[t]{2}{*}{ Odds Ratio } & \multicolumn{2}{|c|}{ 95\% Confidence Interval } & \multirow[t]{2}{*}{ p-value } \\
\hline & & Lower & Upper & \\
\hline Age ( $\geq 40$ vs ref: $<40 y r s$ ) & 1.448 & 0.796 & 2.634 & 0.226 \\
\hline Female (vs ref: Male) & 0.882 & 0.339 & 2.298 & 0.798 \\
\hline Caucasian (vs ref: Chinese) & 0.980 & 0.536 & 1.793 & 0.949 \\
\hline Complaints ( $\geq 2$ vs ref: $I$ ) & $0.44 I$ & 0.202 & 0.965 & 0.040 \\
\hline Prior Treatment ( $\mathrm{Y}$ vs ref: $\mathrm{N})$ & 2.914 & 1.586 & 5.354 & 0.001 \\
\hline Anxious ( $Y$ vs ref: $N$ ) & 0.628 & 0.338 & 1.166 & 0.141 \\
\hline Rejuvenation ( $\mathrm{Y}$ vs ref: $\mathrm{N}$ ) & 2.660 & 1.215 & 5.826 & 0.014 \\
\hline Vanity Score (High $\geq 10$ times vs ref: Low $<10$ times/day) & 0.417 & 0.121 & 1.434 & 0.165 \\
\hline Expectation (Instant vs ref: Progressive) & 1.924 & 1.054 & 3.512 & 0.033 \\
\hline Self Esteem (Positive vs ref: Neutral or Negative) & 0.654 & 0.315 & 1.360 & 0.256 \\
\hline
\end{tabular}

\section{Discussion}

Our study is the first ever conducted to examine patients' characteristics and behavior in seeking out physicians providing non-invasive procedures in an Asian context. Furthermore, this is the first study to demonstrate that having attended an appointment, the overwhelming majority of patients proceed with treatment $(88.5 \%)$. The majority of patients who sought consultation were female, had multiple clinical concerns and had received prior aesthetic treatments. Notable was the high frequency of rejuvenation, rather than correction or aggressive changes in physical appearance as the clinical outcome goal.

Singapore, with a population of 5.6 million in 2018, has over 4 million citizens or permanent residents, out of which $76 \%$ are of Chinese race/ethnicity, ${ }^{17}$ but over $51 \%$ of this study population are Caucasian providing a unique opportunity to understand differences in characteristics and motivations of Chinese and Caucasian patients who seek minimally invasive facial aesthetic procedures. Chinese patients in our study were overrepresented in the younger age group, and more commonly seek correction rather than rejuvenation as a goal of aesthetic procedures. Although it should be noted that rejuvenation as a goal remains the more common aesthetic goal for both Chinese and Caucasian patients and across all age groups, the need for "correction" or more obvious changes was more common among Chinese patients. Our analyses also showed that Caucasian as compared to Chinese race was a significant predictor for proceeding with the recommended treatments, however, this was no longer statistically significant after adjusting for other factors. Nevertheless, this comparison has never been described 
in prior literature and may represent underlying differences in attitudes and behavior between these two populations. Finally, our study identified several predictors of proceeding with treatment on adjusted analyses. Powerful predictors identified included a treatment goal of rejuvenation rather than correction, an expectation of seeing an immediate result, and prior treatment with a surgical or nonsurgical aesthetic procedure. Understanding these factors can inform the consultation process and can help guide the direction and formulation of the treatment plan. For instance, Chinese patients appear to seek more "corrective" changes, suggesting that these patients may focus on more aggressive procedures.

The Asian region is predicted to have the greatest increase in demand for aesthetic procedures and yet studies amongst Asian populations are lacking. Our study is particularly timely as this is set in a multi-racial population of Singapore that can inform differences in attitudes between Chinese and Caucasian patients. Furthermore, our focus on non-invasive facial aesthetic procedures is important given that multiple surveys now show that noninvasive facial aesthetic procedures are in much greater demand that plastic surgery ${ }^{18}$ where the top 5 non-invasive aesthetic treatments are 6-fold more common than all cosmetic surgical procedures combined. As demand for non-invasive procedures is expected to continue, greater understanding on how demographics and clinical characteristics influence consultation and treatment can improve the consultation and treatment planning processes. This study reveals several unique and important clinical findings regarding patients who seek and receive non-surgical facial aesthetic treatments.

Prior studies have not examined the frequency with which patients proceed with treatment after consultation. Almost $90 \%$ of patients consulting proceed with the recommended treatment highlighting the critical importance of the initial consultation. In addition, patients may have already researched the attributes of the treatment, clinic and treating physician, as was apparent from a prior study where physician reputation was identified as one of the most important guiding factors for clinic selection. ${ }^{15}$

Other findings included a low frequency of high vanity scores, and the dominance of intrinsic rather than extrinsic motivators for proceeding with treatment. Patient-reported self-esteem was also positive in over $75 \%$ of patients who sought consultation. Together, these characteristics suggest that patients who seek non-surgical facial aesthetic procedures appear to have a natural clinical outcome in mind. Perhaps patients wanting marked changes in physical appearance, or those frequently checking in a mirror may pursue plastic surgery, rather than minimally invasive procedures. Unfortunately, no research has tracked patients across the interface of these two services that provide assistance in improving appearance but using different techniques.

These attitudes towards non-invasive procedures may potentially translate to more realistic expectations and potentially higher patient satisfaction than motivations that aim for more aggressive changes in physical appearance. $^{19}$

\section{Racial Differences in Motivations for Treatment}

Our analyses examining race and differences in attitudes towards treatment demonstrate a complex interplay of factors that should be considered during the consultation and treatment process. These differences should be taken into account during the consultation process as expectations and goals may differ between various demographic groups.

Although rejuvenation was a more common treatment goal among all patients, Chinese patients more commonly sought correction as a goal that this is more common among Chinese, and the observation that younger patients are more likely to be Chinese suggest a higher emphasis on physical features, or more openness to aesthetic procedures among this racial group as compared to Caucasians. These findings suggest that for Chinese patients, the consultation and treatment plan may focus on a more aggressive approach towards physical transformation as compared to Caucasian patients. These are consistent with recent statistics which suggest that aesthetic procedures are predicted to increase the most in the Asian region. ${ }^{20}$ Furthermore, the aesthetic surgery market in Asia is already one of the highest in the world today. ${ }^{21}$

\section{Factors Associated with Proceeding with Treatment}

Although on initial analyses, Caucasian patients were more likely to proceed with recommended treatments, the difference was no longer statistically significant on multivariate analyses. Indeed, the apparent increased likelihood of Caucasians to proceed with treatment was partially 
explained by a higher proportion of them having had prior procedures, as this likely results in a greater familiarity and comfort with treatments. Indeed, the importance of familiarity is supported by the multivariate modeling where racial origin was no longer statistically significant when prior treatment was added into the model. Patients who have had prior treatments regardless of race, age or gender, were close to three times more likely to proceed with the treatment plan. Altogether, racial origin was no longer a significant predictor of proceeding with treatment after taking into account prior NSFA procedures.

Interestingly, age and gender did not modify the likelihood for proceeding with treatments as these did not show differential effects. This suggests that regardless of age and gender, a high proportion proceed with the recommended treatment, again consistent with patients' having done their own research on clinic, physician and treatment prior to making an appointment to attend the clinic. There was also no differential effect of intrinsic versus extrinsic motivations, suggesting that this did not impact a patient's likelihood to proceed with treatment. Self-reported selfesteem also did not modify a patient's likelihood to proceed with treatment as a high rate of treatment was seen regardless of level of self-esteem reported.

Findings in our study could provide unique insights into patients that seek such treatments. Natural rejuvenation rather than a goal of "correction" was a dominant motivation and a significant predictor for proceeding with treatment. It is possible that patients seeking corrective procedures are more likely to proceed with surgical rather than non-surgical procedures. As information for potential explanatory factors for not proceeding with treatment (including why a preference for correction was associated with non-treatment) was not collected, this area of research deserves further study. On the other hand, our finding that patients with a goal of natural rejuvenation were more likely to proceed with NSFA is consistent with Sobanko's results - that more than half of patients sought "moderate" improvements in clinical appearance, which the authors assessed to be realistic. ${ }^{15}$ This finding is reassuring given that a systematic review, albeit in the plastic surgery literature, identified poor patient outcomes in patients with unrealistic expectations (including those who were identified to be "demanding" and unsatisfied with prior procedures). ${ }^{22-24}$

An expectation of seeking immediate results was a significant predictor of proceeding with treatment. This suggests that patients who do proceed with aesthetic treatments have an expectation that results are immediately visible. Although an immediate result may be feasible in certain treatments, such as with botulinum toxin and dermal fillers, this is not universally applicable with laser procedures, non-invasive skin tightening or collagenbuilding procedures which yield gradual, progressive results. These findings emphasize the importance of caution and counseling as aiming for immediate improvement may be unrealistic.

Finally, after adjusting for other factors, a high number of patient complaints were a statistically significant predictor of non-treatment. These patients were over $55 \%$ less likely to proceed with treatment after the consultation process. This finding suggests that patients who present a high number of complaints may either have unrealistic expectations of non-invasive treatment outcomes or may be unsure on aspects of their facial concerns that they would like to address first. That this could be partially due to higher expectations is consistent with vanity score as a factor associated with non-treatment on unadjusted analysis. It is reassuring that these patients are more careful in deciding whether to proceed with the treatment, but there is also the possibility that they may have sought treatment elsewhere, including seeking plastic surgery, as non-invasive procedures may not address their concerns. Patients who express a high number of complaints and/or present with a high vanity score should be counselled thoroughly during the consultation process in order to ensure that their expectations are clarified.

About $12.5 \%$ of patients did not proceed with any aspect of the recommended treatment plan, their reasons for not proceeding may have included dislike of the treatment options, or cost. Unfortunately, the reasons for not proceeding were not available as these patients never returned to the clinic. Additional studies are required to understand why patients decide not to proceed with treatment after consultation.

\section{Strengths and Weaknesses}

This study has several strengths. It examines an extensive list of clinical, demographic, and behavioral characteristics among patients who seek and receive non-surgical facial aesthetic treatments. Furthermore, in contrast to prior studies, this study included a one-year follow-up and evaluated predictors of actual treatment. ${ }^{14,15}$

Our study demonstrates the benefits of a clinic database for audit and research purposes. A retrospective study design using existing databases is recognized to be 
a powerful and efficient methodology for conducting clinical research as stated by prior publications. ${ }^{25,26}$ In the future, it would be valuable to have an agreed core data set collected in several cosmetic medicine clinics to support multi-practice collaborative projects of practice utilization and clinical outcomes. Another limitation is the lack of a validated questionnaires addressing patient expectations; we used the questions used routinely in our clinical practice but further studies to develop generic, psychometrically tested questionnaires for use in multi-center research are warranted.

As the study includes data from a single clinic, its generalizability to other clinics with a different case mix is limited. Furthermore, our study is set in Singapore, a country with one of the highest GDP per capita in the world and where socio-economic factors may be less of a consideration as compared to other countries. However, there is a wide range of socio-economic status in the country as shown by its high-income inequality or GINI coefficient as compared to other OECD countries. ${ }^{27}$

\section{Conclusions}

Despite the widespread popularity of NSFA procedures, very little is known about patients seeking these treatments. No prior study has evaluated racial differences in demographic, clinical and motivational characteristics as factors that influence proceeding with a treatment plan. This study highlights the profound importance of recognizing differences in Chinese and Caucasian patients, in regards to their attitudes and behavior as these should be taken into account during the initial consultation session. For example, a higher proportion of Chinese patients seek a "corrective" or more aggressive outcome, and may need specific attention towards tempering expectations. Our findings further suggest that although differences in attitude may exist among Chinese and Caucasian patients, there appears to be no significant difference in likelihood to proceed once other explanatory factors, such as prior procedures have been adjusted for.

As our findings suggest that patients are very open and ready to receive treatment once they initiate a discussion with a physician, caution needs to be taken so that patients have time to thoughtfully consider the treatment plan. This is especially the case among patients who express a high number of clinical concerns, and those who report a high vanity score. The "cooling off" period may be of value for patients to step back and assess the recommended treatment plan. Our findings demonstrate that patients are "self-correcting" or "self-adjusting" in that those who proceeded with more comprehensive care did so in a deliberate manner without rushing into the treatment plan. Our research also identified that patients who proceed with NSFA treatments express a goal of natural rejuvenation; a reassuring finding given that patients with more realistic expectations are potentially more likely to achieve their desired aesthetic outcomes.

Finally, this study demonstrates the importance of developing a database in the field of aesthetic medicine and plastic surgery. Such a comprehensive database is potentially a powerful tool that can be harnessed to garner new, clinical insights that may improve clinical care and clinical outcomes.

\section{Disclosure}

The authors report no conflicts of interest for this work and declare that they have no known competing financial interests or personal relationships that could have appeared to influence the work reported in this paper.

\section{References}

1. American Society for Aesthetic Plastic Surgery.Cosmetic surgery national data bank statistics. Aesthetic Surg J. 2018;35(suppl 2):1-24.

2. Atiyeh BS, Rubeiz MT, Hayek SN. Aesthetic/Cosmetic surgery and ethical challenges. Aesthetic Plast Surg. 2008;32(6):829-839. doi:10.1007/s00266-008-9246-3

3. Swami V, Chamorro-Premuzic T, Bridges S, Furnham A. Acceptance of cosmetic surgery: personality and individual difference predictors. Body Image. 2009;6(1):7-13. doi:10.1016/j.bodyim.2008.09.004

4. Brown A, Furnham A, Glanville L, Swami V. Factors that affect the likelihood of undergoing cosmetic surgery. Aesthetic Surg J. 2007;27 (5):501-508. doi:10.1016/j.asj.2007.06.004

5. Swami V, Campana AN, Ferreira L, Barret S, Harris AS, Tavares Mda C. The acceptance of cosmetic surgery scale: initial examination of its factor structure and correlates among Brazilian adults. Body Image. 2011;8(2):179-185. doi:10.1016/j.bodyim.2011.01.001

6. Furnham A, Levitas J. Factors that motivate people to undergo cosmetic surgery. Can J Plastic Surg. 2012;20(4):e47-e50. doi:10.1177/229255031202000406

7. Brunton G, Paraskeva N, Caird J, et al. Psychosocial predictors, assessment, and outcomes of cosmetic procedures: a systematic rapid evidence assessment. Aesthetic Plast Surg. 2014a;38 (5):1030-1040. doi:10.1007/s00266-014-0369-4

8. Shridharani SM, Magarakis M, Manson PN, Rodrigues ED. Psychology of plastic and reconstructive surgery: a systematic clinical review. Plast Reconstr Surg. 2010;126(6):2243-2251. doi:10.1097/PRS.0b013e3181f445ae

9. Ishigooka J, Iwao M, Suzuki M, Fukuyama Y, Murasaki M, Miura S. Demographic features of patients seeking cosmetic surgery. Psychiatry Clin Neurosci. 1998a;52(3):283-287. doi:10.1046/ j.1440-1819.1998.00388.x

10. Nami.org. NAMI: National Alliance on Mental Illness mental health by the numbers. [Online]; 2017. Available from: https://www.nami. org/Learn-More/Mental-Health-By-the-Numbers. Accessed April 14, 2017. 
11. Ende KH, Lewis DL, Kabaker SS. Body dysmorphic disorder. Facial Plast Surg Clin North Am. 2008;16(2):217-223. doi:10.1016/j. fsc. 2007.11.012

12. Von Soest T, Kvalem IL, Wichstrom L. Predictors of cosmetic surgery and its effects of psychological factors and mental health: a population based follow up study among Norwegian females. Psychol Med. 2012;42:617-626. doi:10.1017/S0033291711001267

13. Schofield M, Hussain R, Loxton D, Miller Z. Psychosocial and health behavioral covariates of cosmetic surgery: women's health Australia study. J Health Psychol. 2002;7(4):445-457. doi:10.1177/135910 5302007004332

14. Maisel A, Waldman A, Fudan K, et al. Self-reported patient motivations for seeking cosmetic procedures. JAMA Dermatol. 2018;154 (10):1167-1174. doi:10.1001/jamadermatol.2018.2357

15. Sobanko JF, Taglienti AJ, Wilson AJ, et al. Motivations for seeking minimally invasive cosmetic procedures in an academic outpatient setting. Aesthetic Surg J. 2015;35(8):1014-1020. doi:10.1093/asj/sjv094

16. Rosner B. Fundamentals of Biostatistics.7th ed.Boston: Brooks/Cole, Cengage Learning; 2011.

17. Singapore Department of Statistics (DOS). Population; 2018. Available from: https://www.singstat.gov.sg/-/media/files/publica tions/population/population2018.pdf. Accessed May 9, 2017.

18. American Society of Plastic Surgeons. National plastic surgery statistics; 2018. Available from: https://www.plasticsurgery.org/documents/News/ Statistics/2018/plastic-surgery-statistics-report-2018.pdf. Accessed May 12, 2017.

19. Waljee J, McGlinn EP, Sears ED, Chung KC. Patient expectations and patient-reported outcomes in surgery: a systematic review. Surgery. 2014;155(5):799-808. doi:10.1016/j.surg.2013.12.015
20. South Morning China Post. China's cosmetic surgery market to explode in the next few years, analysts say, Online; February 15, 2016. Available from: https://www.scmp.com/business/companies/ article/1913191/chinas-cosmetic-surgery-market-explode-next-fewyears-analysts. Accessed May 5, 2017.

21. Statista. Market size of the aesthetic medical and surgical industry in China from 2012 to 2019, with a forecast until 2023; October 19, 2020. Available from: https:/www.statista.com/statistics/1089021/chinamedical-aesthetics-industry-market-size/. Accessed May 8, 2017.

22. Block AR, Sarwer DB, editors. Presurgical Psychological Screening: Understanding Patients, Improving Outcomes. American Psychological Association Publishing; 2013.

23. Castle D, Phillips K, Dufresne R. Body dysmorphic disorder and cosmetic dermatology: more than skin deep. $J$ Cosmet Dermatol. 2004;3(2):99-103. doi:10.1111/j.1473-2130.2004.00105.x

24. Herruer JM, Prins JB, Van Heerbeek N, Verhage-Damen GW, Ingels KJ. Negative predictors for satisfaction in patients seeking facial cosmetic surgery: a systematic review. Plast Reconstr Surg. 2015;135(6):1596-1605. doi:10.1097/PRS.0000000000001264

25. Raghupathi W, Raghupathi V. Big data analytics in healthcare: promise and potential. Health Inf Sci Syst. 2014;2:3. doi:10.1186/2047-2501-2-3

26. Zhu VZ, Tuggle CT, Au AF. Promise and limitations of big data research in plastic surgery. Ann Plast Surg. 2016;76(4):453-458. doi:10.1097/SAP.0000000000000750

27. Economist.com; 2017. Available from: http://www.economist.com/ news/special-report/21657611-we-dont-them-much-rich-are-alwaysus. Accessed May 6, 2017.

\section{Publish your work in this journal}

Clinical, Cosmetic and Investigational Dermatology is an international, peer-reviewed, open access, online journal that focuses on the latest clinical and experimental research in all aspects of skin disease and cosmetic interventions. This journal is indexed on CAS.
The manuscript management system is completely online and includes a very quick and fair peer-review system, which is all easy to use. Visit http://www.dovepress.com/testimonials.php to read real quotes from published authors. 